\title{
Measurement of long-range steric repulsions between microspheres due to an adsorbed polymer
}

\author{
R. J. Owen, ${ }^{*}$ J. C. Crocker, ${ }^{\dagger}$ R. Verma, ${ }^{\dagger}$ and A. G. Yodh \\ Department of Physics and Astronomy, University of Pennsylvania, 209 South 33rd Street, Philadelphia, Pennsylvania 19104
}

(Received 7 October 2000; published 11 June 2001)

\begin{abstract}
We have measured the interparticle potential between pairs of micron-sized silica spheres induced by adsorbed polyethylene oxide polymer using a line-scanned optical tweezer. We found this long-range steric repulsion to be exponential over the range of energies $\left(0.1 k_{B} T-5 k_{B} T\right)$ and polymer molecular weights (452000-1580000) studied, and that the potential scaled with the polymer's radius of gyration $R_{G}$. The potential's exponential decay length was about $0.6 R_{G}$ and its range was about $4 R_{G}$, although both parameters varied significantly from one pair of spheres to another. The potential's exponential prefactor was greater than mean-field predictions.
\end{abstract}

DOI: 10.1103/PhysRevE.64.011401

PACS number(s): 61.25.Hq, 82.70.Dd, 68.43.- h

\section{INTRODUCTION}

The adsorption of polymers onto the surfaces of colloidal particles can stabilize or destabilize the suspension, depending on polymer surface coverage as well as on polymerpolymer and polymer-particle interactions [1]. If, for example, particles collide with partially covered surfaces then free ends or loops of polymer chains adsorbed to one particle may stick to bare patches on the other, forming bridges and causing flocculation. On the other hand, if particle surfaces are fully covered with polymer, as shown in Fig. 1, then the suspension can be stabilized because of the steric repulsions arising between the adsorbed layers of neighboring particles. Controlling such colloidal stabilization requires a microscopic understanding of the adsorbed polymer layer and its effects on the thermal-scale interactions between colloidal particles.

Our experiments employ optical tweezers and video microscopy, techniques that have recently been used to probe the microscopic interactions in a variety of interesting colloidal systems [2-7]. In this contribution, we investigate the pair interaction potential for a colloidal model system stabilized by adsorbed polymer. We choose relatively monodisperse polyethylene oxide (PEO), $\left(\mathrm{CH}_{2} \mathrm{CH}_{2} \mathrm{O}\right)_{n}[8-19]$, as our polymer species and silica microspheres as our particle species. PEO is a commercially important, water-soluble linear homopolymer that adsorbs onto silica under appropriate chemical conditions. Our measurements provide quantitative molecular-weight-dependent information about the structure of the adsorbed polymer layer and the polymer-induced repulsion between colloidal particles on the thermal energy scale. Our observations about the form of the potential confirm recent mean-field and scaling theories [20-22], and are comparable to recent measurements of forces between liquid interfaces with a different adsorbed polymer [23]. To the best

\footnotetext{
*Present address: H. H. Wills Physics Laboratory, University of Bristol, Tyndall Avenue, Bristol, BS8 1TL, UK.

${ }^{\dagger}$ Present address: Applied Physics Department, California Institute of Technology, Mail Code 128-95, Pasadena, CA 91125.

${ }^{\ddagger}$ Present address: Unilever Research, 45 River Road, Edgewater, NJ 07020.
}

of our knowledge, the present optical tweezer experiments are the first to directly probe steric contributions of molecules on the surfaces of interacting colloidal particles.

Our approach complements previous studies of steric interactions in model systems based on different experimental methodologies. Several techniques have been used to extract the effective thickness of a layer of polymer adsorbed onto the surface of a colloidal particle. Some methods, for example, measure a change in the hydrodynamic diameter of the particles using light scattering, viscometry, or sedimentation $[11,17]$. More detailed microscopic information about the polymer layer is derived with methods of greater complexity. For example, several techniques have been developed and applied to extract mean force profiles between sterically stabilized surfaces. The surface force apparatus (SFA) was employed to measure the forces between mica surfaces covered with adsorbed PEO $[14,15,19]$ and in other systems [16,24,25]. Atomic force microscopy (AFM) was modified to study this problem by attaching a large colloidal particle to the AFM tip and then measuring the force experienced by the particle as it approaches a flat plate; in this case both surfaces were exposed to solutions of PEO $[8,9]$. Neutron reflectivity [18] and total internal reflection fluorescence $[12,13]$ have also been used to study PEO interactions. Finally and perhaps most relevant to the present paper, magnetic emulsion force-distance and disjoining pressure measurements have been employed [23] to study adsorbed polymer-induced repulsion between liquid surfaces at nearthermal energies.

With the exception of Mondain-Monval et al. [23], the
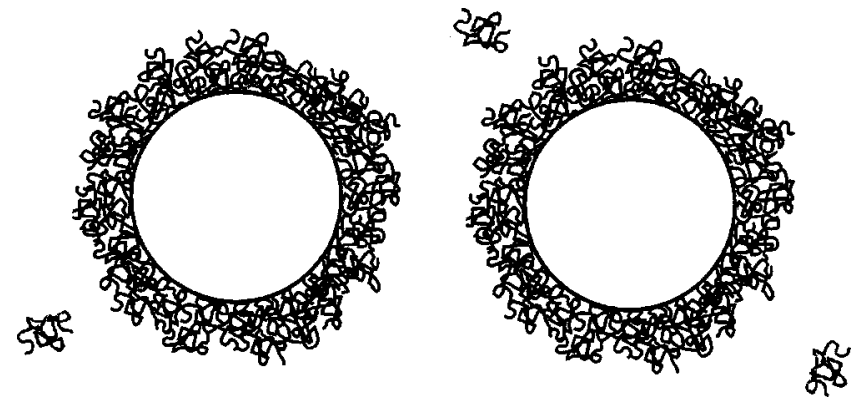

FIG. 1. Schematic of colloidal microspheres coated with adsorbed polymer (drawing not to scale). 
TABLE I. PEO Data.

\begin{tabular}{ccc}
\hline \hline $\begin{array}{c}\text { Molecular weight } \\
M_{w}\end{array}$ & $\begin{array}{c}\text { Polydispersity } \\
M_{w} / M_{n}\end{array}$ & $\begin{array}{c}\text { Radius of gyration } \\
R_{G}(\mathrm{~nm})\end{array}$ \\
\hline 1580000 & 1.06 & 74.7 \\
993000 & 1.08 & 65.7 \\
755000 & 1.07 & 52.3 \\
452000 & 1.06 & 37.2 \\
\hline
\end{tabular}

microscopic techniques primarily provide information about polymer structure or density within a strongly compressed polymer layer. While these measurements are needed to understand the full structure of the adsorbed layer, the compression energies involved are often hundreds of $k_{B} T$, and thus do not correspond to the Brownian collisions seen in actual colloids. Furthermore, Ruths et al. have seen irreversible changes in the polymer layer after the first SFA compression cycle [25] and layer changes have also been seen with AFM [9].

In the present work, two colloidal particles are held in a line-scanned optical tweezer, and are driven together only by their Brownian motion. Thus, the polymer layer is not compressed significantly and our observations are directly applicable to real colloidal systems. We find clear differences between measurements with and without added polymer. In the presence of PEO (1) the range of the repulsive core increases and (2) the repulsion becomes softer, having an exponential form. As we varied the radius of gyration of the polymer, $R_{G}$, we found that both the range and decay length of the potential scaled. The interaction's exponential decay length was $\approx 0.6 R_{G}$, and its range was $\approx 4 R_{G}$. The overall strength of the interaction we observed for PEO adsorbed to silica was comparable to recent measurements employing PVA-Vac adsorbed on an air/water interface [23].

\section{EXPERIMENT}

Our mixed polymer/colloid samples consisted of roughly $10^{-6}$ weight fraction silica microspheres suspended in a $0.10 \%$ by weight PEO solution. The mixture was buffered at $p \mathrm{H} 8.0$ by $10 \mathrm{mM}$ TRIS (Hydroxymethyl-aminomethane), and $1 \mathrm{mM}$ Sodium azide and $1 \mathrm{mM}$ EDTA (Ethylenediaminetetraacetic acid) were added to inhibit bacterial growth. The silica particles were $1.1 \mu \mathrm{m}$ diameter and were obtained from Bangs Laboratories. Since we measured individual pairs of particles, only very low particle concentrations were required. Four different molecular weight PEO samples were obtained from Polymer Laboratories. The polymer samples are relatively monodisperse, and are intended as chromatographic standards. The molecular weight, polydispersity and radius of gyration $R_{G}$ were determined by Polymer Laboratories from light scattering data, and are summarized in Table I. At $0.10 \%$, the background polymer concentration was well below the overlap concentration $C^{*}$, and the low particle concentration ensured that there was always an excess of polymer in solution relative to that adsorbed.

The surface of the silica has silanol groups, $\mathrm{SiOH}$, which can dissociate according to an acid-base equilibrium [26];

$$
\mathrm{SiOH} \leftrightarrow \mathrm{SiO}^{-}+\mathrm{H}^{+}
$$

The fraction of dissociated surface groups and hence the surface charge on the particles is highly dependent on the $p \mathrm{H}$ $[1,26]$. It is critical to use a $p \mathrm{H}$ buffer when studying $\mathrm{PEO}$ and silica in an aqueous environment. Indeed, irreproducibility in the adsorption rate of PEO on glass has been reported in experiments that did not control $p \mathrm{H}$ [9]. Thus all our solutions were buffered at $p \mathrm{H} 8.0$ using $10 \mathrm{mM}$ TRIS. This also ensured that the charge screening length was approximately $5 \mathrm{~nm}$, much smaller than the thickness of the adsorbed polymer layer.

Although water is a good solvent for PEO at room temperature, the solution behavior of PEO in water is not straightforward [27-29]. Special care was taken to minimize chemical degradation of the PEO (particularly due to UV light exposure), and to minimize the presence of bacteria in the suspension that could lead to protein and lipid adsorption on the silica surface [26]. The dry polymer was kept refrigerated and the PEO solutions were kept in the dark and at room temperature during the mixing stages so that the solubility would be that of the final mixture. The PEO was allowed to dissolve for $48 \mathrm{~h}$, then the silica spheres were added to the solution, which was allowed 24 more hours to reach equilibrium. Since the polymer was less stable in solution than dry, no measurements were made with polymer that had been in solution for more than a week. All equipment used with the solutions was autoclaved and the water was deionized, autoclaved and $0.2 \mu \mathrm{m}$ filtered. All of the above procedures were found to be necessary to achieve consistent interaction potentials. This presumably occurs concurrently with full adsorption coverage on the silica particle surface.

The details of the line-scanned optical tweezer and microscopy are described in previous works [2,3,6,7]. Briefly, the two colloidal spheres freely diffuse along a line in the optical trap. The particles are strongly confined in the two other dimensions, while a gentle, nearly harmonic optical potential along the line ensures that the particles spend most of their time near the central portion of the optical trap. Thus the particles are free to explore their equilibrium energy landscape, and we obtain good statistics near contact that is critical for accurate interaction measurements.

Our approach is to measure the center-center separation of the two diffusing particles and then extract their interaction potential from the probability of finding them at a given separation. The motion of two particles in the optical trap is recorded for 25-40 min (at 30 frames a second) using a CCD camera and a video cassette recorder. Images were first digitized using NIH Image and then analyzed using custom programs written in the language IDL. The analysis locates the centroids of the overlapping, diffraction-blurred sphere images as discussed elsewhere [2,3,7]. By constructing a histogram of the center-center separations on the roughly $10^{5} \mathrm{im}-$ ages, we can accurately estimate the probability $P(r)$ of finding the particles separated by $r$. The system's Helmholtz free energy $F(r)$ (equivalent to the pair interaction potential) 


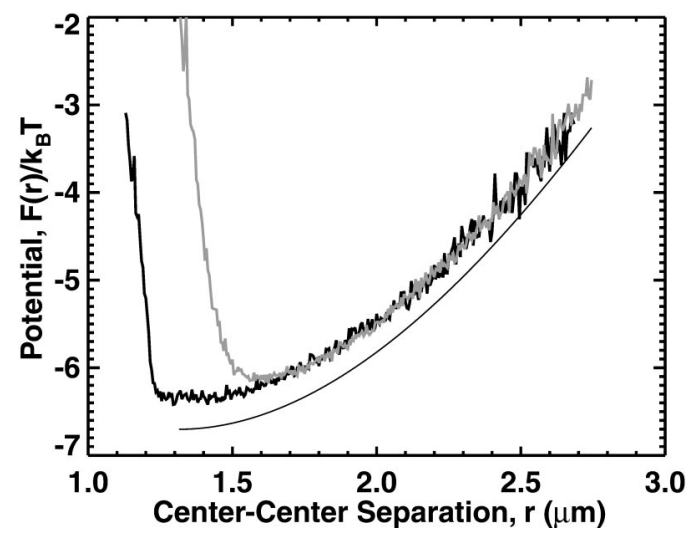

FIG. 2. Buffer potential (dark curve) and the potential measured with PEO in solution (light curve). The effect of the polymer layer is seen in the shift of the strongly repulsive part of the PEO solution potential to larger particle separations, and in the comparatively softer decay of the PEO solution potential. At large separations, both potentials are dominated by the optical trap; modeled by a fit to the buffer potential (offset curve).

can then be determined up to an additive offset by using the Boltzmann relation, $P(r) \sim \exp \left[-F(r) / k_{B} T\right]$.

One potential curve is measured for solutions containing PEO and another for a buffer solution without polymer but under otherwise identical conditions. By subtracting the buffer potential from the potential for solutions containing PEO, we isolate the effects of the adsorbed polymer layer from the other contributions to interparticle potential, e.g., electrostatic effects, Van der Waals forces, etc. This subtraction relies upon the harmonic form of the particles' potential energy along the optical line trap. The potential we measure is technically the potential averaged over time and the equilibrium orientations of both beads. This should be kept in mind since the polymer layer on both beads cannot be perfectly spherically symmetric. This should cause no problem, however, when comparing our results with the predictions of mean-field theories.

\section{RESULTS}

Figure 2 shows an example of the uncorrected interparticle potential energy measured between two microspheres with and without PEO in solution. The potential without PEO exhibits a sharp rise at small separations due to shortrange electrostatic repulsion between the spheres charged surfaces. At long range, the potential has a nearly parabolic form due to the two spheres confinement in the parabolic optical trap. Differences in the interaction potential with adsorbed PEO are quite evident; the repulsive core of the potential is shifted to larger separations and this repulsive regime exhibits a softer decay than for the bare potential.

To isolate the effects of the adsorbed polymer from those due to the optical trap, we can simply take the difference of the two potentials with and without polymer. In order to avoid adding unnecessary noise to the result, we actually subtract a fit to the buffer potential, such as the smooth curve shown in Fig. 2. Figure 3(a) shows the result of the subtraction on a linear scale. The strong repulsion at small separa-
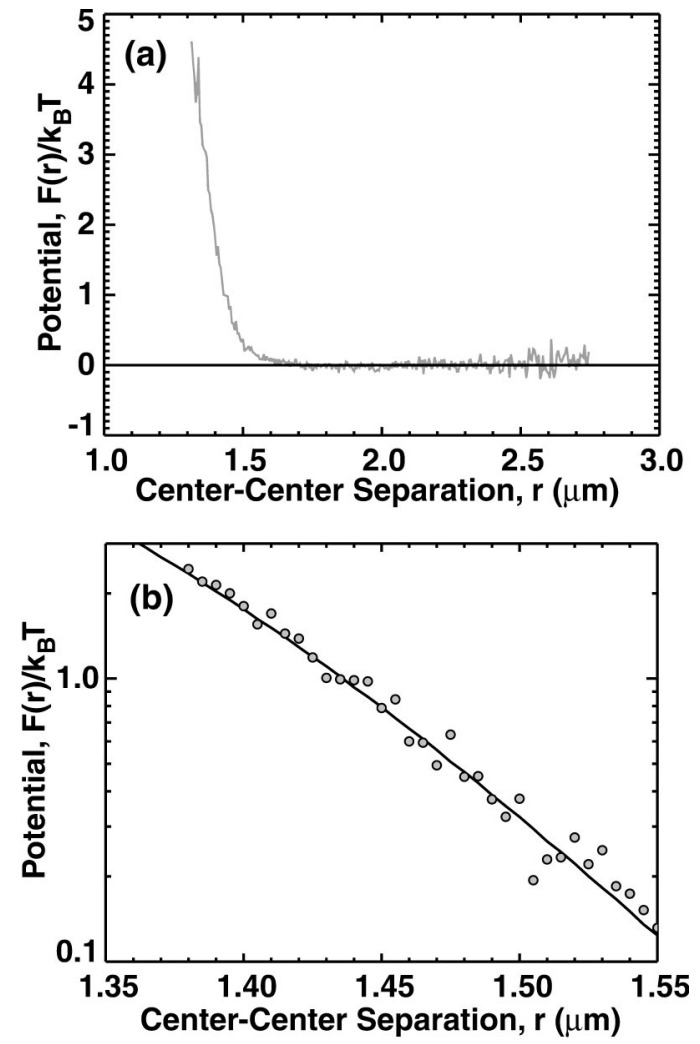

FIG. 3. Background-subtracted interaction potential; the smooth fit to the buffer has been subtracted from the PEO solution potential to derive a potential due solely to the adsorbed polymer. (a) Potential shown on linear scale, (b) Initial section of the potential shown on a semilog scale. The decay of the repulsion is well modeled by the line, which shows a simple exponential fit that has been corrected for the effects of instrumental resolution.

tions (e.g., up to $5 k_{B} T$ ) decays to zero as the particle separation increases. This potential is replotted in Fig. 3(b) to display its nearly exponential character.

To begin, we modeled the interaction potential with an exponential of the form

$$
\frac{F(r)}{k_{B} T}=\exp \{-[r-2(a+\delta a)] / \lambda\}
$$

where $a$ is the microsphere radius, $\lambda$ is the exponential decay length, and the potential equals $k_{B} T$ at a separation $r=2(a$ $+\delta a$ ). In this form, $2 \delta a$ approximates the range of the steric repulsion and we can intuitively identify $\delta a$ with the effective thickness of the adsorbed polymer layer. For our fits, we took both $\lambda$ and $\delta a$ to be free adjustable parameters, while constraining the particle diameter, $2 a=(1.134$ $\pm 0.015) \mu \mathrm{m}$.

In order to make quantitative fits to our data, we must take the finite spatial resolution of our instrument into account [3]. Errors in the measured center-center separation are caused by camera noise as well as small out-of-plane motions of the microspheres. Such errors cause the observed probability distribution $P(r)$ to resemble the expected $P(r)$ blurred (convolved) with a Gaussian. To take such blurring 

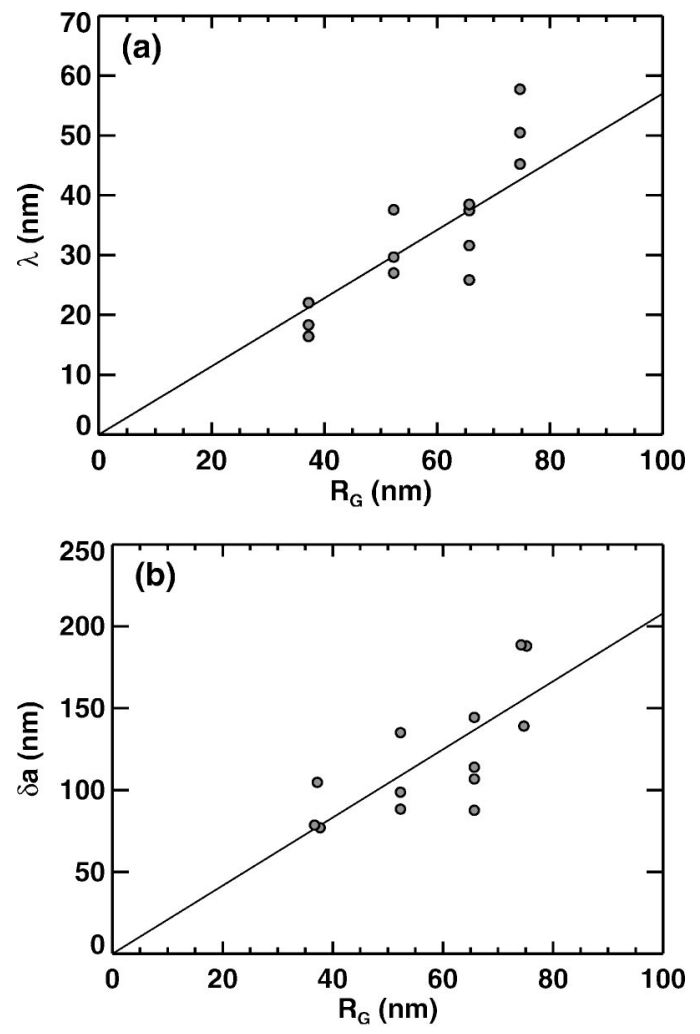

FIG. 4. (a) Exponential decay length $\lambda$ versus $R_{G}$ for the four PEO samples (see Table 1). Several independent measurements were made for each value of $R_{G}$, which are shown as separate points on the plot. A linear fit constrained to go through the origin gives $\lambda=0.57 R_{G}$, and is shown for comparison with the data. (b) The increase in apparent particle radius, $\delta a$, as a function of $R_{G}$; a linear fit gives $\delta a=2.1 R_{G}$, and is shown in the figure.

into account during modeling, we can simply exponentiate the model potential to yield $P(r)$, numerically convolve it with a Gaussian, and finally take a logarithm to return an appropriately "blurred" model potential. Such blurred potential models can then be fit to the experimental data with a numerical least-squares algorithm. We independently determine the instrumental resolution $\sigma_{\text {blur }}$ for these experiments by fitting the buffer-only potentials, which yields $\sigma_{\text {blur }}$ $=(35 \pm 2) \mathrm{nm}$.

We used this blurring technique to fit Eq. (2) to interaction potentials with added PEO, a typical example is shown in Fig. 3(b). We see that the shape of the curve fits quite well, demonstrating that the independently determined $\sigma_{\text {blur }}$ is able to explain the observed small deviation from an exponential. Indeed, we find that all of our measured data is consistent with a purely exponential form for the $k_{B} T$-scale steric repulsion due to adsorbed PEO.

We systematically examined the dependence of our observed model parameters $\lambda$ and $\delta a$ on the polymer radius of gyration $R_{G}$. In Fig. 4(a) we plot the exponential decay lengths, $\lambda$. Each point represents an individual pair of microspheres measured in an independently prepared polymer solution. Because of the scatter in the data, a linear fit seemed reasonable as a first approximation; the data does not justify the use of a more complex function. A best linear fit through the origin suggests $\lambda=(0.57 \pm 0.05) R_{G}$ and is shown in Fig. 4(a). The corresponding results for $\delta a$ are shown in Fig. 4(b). We felt it was appropriate to choose the simplest ad $h o c$ fit to the data. A linear fit through the origin yields $\delta a$ $=(2.1 \pm 0.2) R_{G}$.

While we fit to an exponential form as given in Eq. (2), theoretical treatments [20-22] often describe the interaction by

$$
\frac{F(r)}{k_{B} T}=A e^{-(r-2 a) / \lambda},
$$

where $\lambda$ is the decay length as before, and $A$ is a coupling constant describing the strength of the interaction. While we could have fit our data to Eq. (3) just as well, small errors in the fit would have lead to exponentially large errors in A. For comparisons with theory however, our results can be converted to be in terms of $\lambda$ and $A$. Equating the formulas for both models gives simply $A=\exp (2 \delta a / \lambda)$. Combining this with the linear slopes in Fig. 4 suggests that $A=\exp (7.3$ $\pm 0.9)$. This corresponds to the most likely value being $A$ $=850$, with the one standard deviation error placing it in the range $350<A<1800$.

The observed exponential form of the long-range potential is consistent with theory [20-22] and with force experiments on liquid interfaces with adsorbed polymer [23]. Our best estimate of the exponential decay length $\left(\lambda=0.6 R_{G}\right)$ was smaller but comparable to the value observed in the liquid droplet experiments $\left(\lambda=R_{G}\right)$. A mean-field theory [20-22] for adsorbed polymers, predicts that $A=(\pi a / \lambda)$ in the strong adsorption limit. Substituting $a=567 \mathrm{~nm}$ and $\lambda$ $=30 \mathrm{~nm}$, this predicts $A \approx 100$, almost an order of magnitude smaller than our measurement above. By multiplying our result for $A$ by $\left(k_{B} T / \lambda\right)$ we convert our measured exponential prefactor to an exponential force prefactor. In this case we get a force prefactor of $\approx 10^{-10} \mathrm{~N}$, comparable to the film-disjoining-pressure measurements of [23] on a different adsorbed polymer system. Thus we find that the strength of the interaction is higher than mean-field predictions for strongly adsorbed polymers, while it is comparable to measurements carried out on other polymer systems. This discrepancy with theory remains to be explained.

\section{DISCUSSION}

Using the line-scanned optical tweezer we have measured the long-range interaction potential between two silica spheres for buffered solutions with and without adsorbed PEO. The effects of the adsorbed PEO layer were clearly evident. The potential resulting primarily from the PEO tails has been isolated, and was found to be well approximated by an exponential (after accounting for instrumental resolution). The exponential decay lengths were measured for four different molecular weights of PEO between 452000 and 1580000 , and the independent measurements of these decay lengths are largely consistent. On average the decay lengths were found to be approximately 0.6 times the radius of gyration of the polymers used. A number characterizing the increase in apparent size of the particles was also measured. 
The increase in apparent radius of the particle was approximately 2.1 times the radius of gyration of the polymer. We will briefly address two important issues below: measurement scatter and relation to theoretical predictions.

An important observation of the current experiments is the considerable scatter in results for both $\lambda$ and $\delta a$. This scatter was not evident in many measurements of the same particle, but instead was manifest from particle to particle and from sample batch to sample batch. We believe that at least some of the variability may be intrinsic to the PEOsilica system. Differences in adsorbed configurations can result from differences in sample history, aging effects, small heterogeneities in the surface chemistry of the particles, small temperature differences, etc. In a related vein, total internal reflection fluorescence studies of self-exchange and interfacial relaxation in the PEO-water-silica system (buffered at $p \mathrm{H}$ 7.1) [13] have suggested that the polymer in the surface layer may be trapped in extremely long-lived metastable states, and that a subpopulation of the chains are irreversibly attached to the surface. The polymer in the surface layer was affected by entanglements and by large numbers of segment-surface contacts and appeared to be in a glassy state. This work [13] used PEO of molecular weight 33000 and 120000 , whereas for our work the smallest PEO had a molecular weight of 452000 . The effects of quenched-in nonequilibrium states in the surface layer might be expected to increase with molecular weight. We examined the effects of the time the polymer had been in solution and also the time that the polymer had been mixed with the beads (on time scales of order $24 \mathrm{~h}$ ), but no clear correlations were found in either case; nevertheless the relaxation times might be very long. On the other hand, our measurements accurately reflect the actual behavior of suspensions of adsorbed$\mathrm{PEO} /$ silica in real colloidal suspensions, and our conclusions about the form of the potential survive the data scatter.

The most significant result of the paper is that the longrange thermal interaction potential between two colloidal particles in suspension coated with adsorbed polymer exhib- its an approximately exponential repulsion with distance, and that this decay length depends linearly on the radius of gyration of the adsorbed polymer. The background polymer concentration was small (i.e., below the overlap concentration $\mathrm{C}^{*}$ ) and the solvent was good. For these studies we have employed an experimental technique that differs qualitatively from previous methods used to study adsorbed polymers. Clearly, our measurements are not well described by the Gaussian or parabolic forms predicted for other polymer/ colloid systems (e.g., monomer brushes, etc.) [30-32].

The exponential decay length of the potential with separation has been predicted by both mean-field and scaling theory [20-22] to be proportional to $R_{G}$, and is thus consistent with our experiments; the exact constant of proportionality, however, is difficult to calculate without detailed knowledge of polymer adsorption strength and about the polymer concentration profile closer to the wall of the particle. In principle the combination of experiment and theory can be used to characterize these parameters for the adsorbed-PEO/silica system. The methodology we have developed in combination with the scaling theory should enable experimenters to microscopically characterize a much broader range of adsorbed-polymer/colloid systems. Furthermore, since the osmotic pressure between the particles should be dominated by two-point contacts of the monomers, our measurements provide information about monomer density distribution for the polymer tails, far from the particle surface.

\section{ACKNOWLEDGMENTS}

We are delighted to acknowledge extensive discussions about the theory with Albert Johner and Scott Milner. We also thank Russ Composto, Nili Dan, Lyderic Bocquet, Bill Russell, and Randy Kamien for useful discussions. R.J.O. was funded at the University of Pennsylvania by Thouron Scholarship Fund. This work was supported by the NSF through Grant No. DMR-99-71226 and partially by the NSFMRSEC through Grant No. DMR-96-32598.
[1] G.J. Fleer, M.A. Cohen Stuart, J.M.H.M. Scheutjens, T. Cosgrove, and B. Vincent, Polymers at Interfaces (Chapman and Hall, London, 1993)

[2] J.C. Crocker and D.G. Grier, J. Colloid Interface Sci. 179, 298 (1996).

[3] J.C. Crocker, J.A. Matteo, A.D. Dinsmore, and A.G. Yodh, Phys. Rev. Lett. 82, 4352 (1999).

[4] D. Grier, Curr. Opin. Colloid Interface Sci. 2, 264 (1997).

[5] M.T. Valentine, L.E. Dewalt, and H.D. OuYang, J. Phys.: Condens. Matter 8, 9477 (1996).

[6] R. Verma, J.C. Crocker, T.C. Lubensky, and A.G. Yodh, Phys. Rev. Lett. 81, 4004 (1998).

[7] R. Verma, J.C. Crocker, T.C. Lubensky, and A.G. Yodh, Macromolecules 33, 177 (2000).

[8] S. Biggs, Langmuir 11, 156 (1995).

[9] G.J.C. Braithwaite and P.F. Luckham, J. Chem. Soc., Faraday Trans. 93, 1409 (1997); G.J.C. Braithwaite, A. Howe, and P.F.
Luckham, Langmuir 12, 4224 (1996).

[10] M.A. Cohen Stuart and H. Tamai, Langmuir 4, 1184 (1988).

[11] E.C. Cooper, P. Johnson, and A.M. Donald, Polymer 32, 2815 (1991).

[12] Z. Fu and M.M. Santore, Macromolecules 31, 7014 (1998).

[13] Z. Fu and M.M. Santore, Macromolecules 32, 1939 (1999).

[14] J.N. Israelachvili, R.K. Tandon, and L.R. White, J. Colloid Interface Sci. 78, 432 (1980).

[15] J. Klein and P.F. Luckham, Macromolecules 17, 1041 (1984).

[16] T.L. Kuhl, A.D. Berman, S.W. Hui, and J.N. Israelachvili, Macromolecules 31, 8258 (1998).

[17] F. Lafuma, K. Wong, and B. Cabane, J. Colloid Interface Sci. 143, 9 (1991).

[18] E.M. Lee, R.K. Thomas, and A.R. Rennie, Europhys. Lett. 13, 135 (1990).

[19] P.F. Luckham and J. Klein, J. Chem. Soc., Faraday Trans. 86, 1363 (1990) 
[20] G.J. Fleer, J. van Male, and A. Johner, Macromolecules 32, 825 (1999).

[21] G.J. Fleer, J. van Male, and A. Johner, Macromolecules 32, 845 (1999).

[22] A.N. Semenov, J.-F. Joanny, A. Johner, and J. Bonet-Avalos, Macromolecules 30, 1479 (1997).

[23] O. Mondain-Monval, A. Espert, P. Omarjee, J. Bibette, F. Leal-Calderon, J. Philip, and J.-F. Joanny, Phys. Rev. Lett. 80, 1778 (1998).

[24] M. Ruths, H. Yoshizawa, L.J. Fetters, and J.N. Israelachvili, Macromolecules 29, 7193 (1996).

[25] M. Ruths, J.N. Israelachvili, and H.J. Ploehn, Macromolecules 30, 3329 (1997).
[26] R.K. Iler, The Chemistry of Silica (Wiley, New York, 1979).

[27] S. Kawaguchi, G. Imai, J. Suzuki, A. Miyahara, T. Kitano, and K. Ito, Polymer 38, 2885 (1997).

[28] S. Kinugasa, H. Nakahara, N. Fudagawa, and Y. Koga, Macromolecules 27, 6889 (1994).

[29] B. Porsch and L.-O. Sundelöf, Macromolecules 28, 7165 (1995).

[30] P.G. de Gennes, Macromolecules 14, 1637 (1981); 15, 492 (1982).

[31] S.T. Milner, Science 251, 905 (1991).

[32] S.S. Patel and M. Tirrell, Annu. Rev. Phys. Chem. 40, 597 (1989). 